
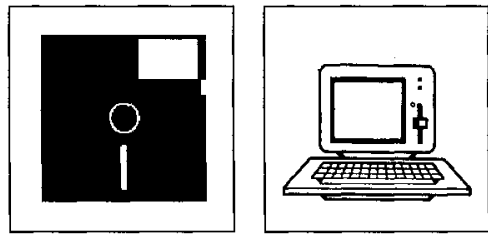

„Echte" Netzwerksoftware: Nicht „nebenbei" zu erledigen

Konsequenzen für den Test von Anwaltssoftware unter "Voll-Last"
Netzwerkfähige Anwaltssoftware

\section{Fazit ...}

Vorliegend wurde der Versuch unternommen, konzeptionelle Grundsätze aus dem Bereich „Netzwerksicherheit” zu erörtern, deren Außerachtlassung schon manchen „abgestürzten” Anwender leider mehr an der EDV als an seinem Programm zweifeln ließ; zusätzlich sollte ein grober Überblick über die Funktionalität zeitgemäßer, netzwerkfähiger Software gegeben werden. Nicht eingegangen werden konnte daher auf die gesamte Palette der programmierbaren Schutzmechanismen, die ein Betriebssystem wie NOVELL „seinen” Programmen anbietet. Es dürfte allerdings deutlich geworden sein, daß „echte” Netzwerksoftware nicht ohne weiteres von einem Wochenendprogrammierer erstellt werden kann, der durchaus ein Gebührenprogramm zustande zu bringen vermag; dies darf angesichts der Qualitätsunterschiede angebotener Anwaltssoftware durchaus einmal ausgesprochen werden. Selbst „Blackbox”-Dateiverwaltungssysteme, die gerne für wirtschaftliche Programmprojekte herangezogen werden, bieten oft von ihren Funktionen her bereits nicht die Möglichkeit, differenzierte Schutzreaktionen im Netz vorzusehen.

Schließlich sei dem Verfasser die Feststellung erlaubt, daß auch Anwaltssoftware in softwaretechnischer Hinsicht eingehender getestet werden sollte, wobei die Überprüfung der Netzwerksicherheit und Arbeitsqualität eines Programmes unter praxistypischer „VollLast” erfolgen müßte; nur der gemeinsame (kollisionäre) Datenzugriff im Netz auf eine praxisgerechte Anzahl von Datensätzen über eine Referenzhardware zeigt, was eine Software tatsächlich leistet.

jurpc.zip - jurpc.zip - jurpc.zip - jurpc.zip - jurpc.zip - jurpc.zip - jurpc.zip

\section{Anmerkung zu KG Berlin, Urteil vom 30. März 1993 (5 U 7655/92)} (jur-pc 11/93, S. 2365, in diesem Heft)

Da der „Clementinen”-Fall des BGH (25.6.1992, I ZR 136/90, GRUR 1992, 858-860) vom KG als Präjudiz diskutiert wird, rechtfertigt sich die Frage, wie man aus einer für Clementinen formulierten Regel etwas für Notebooks gewinnen kann.

Im Clementinen-Fall fehlte es an den beworbenen Clementinen. Ausgesprochen wurde deswegen zunächst ein auf Lebensmittel bezogenes Werbeverbot (für den Fall mangelnden Vorrats). Der BGH hat das als zu weitgehend angesehen und den Unterlassungsanspruch auf den Bereich von Obst und Gemüse begrenzt:

„In der Rechtsprechung des Bundesgerichtshofs ist anerkannt, daß im Interesse eines hinrei"henden Rechtsschutzes gewisse Verallgemeinerungen zulässig sind, sofern auch in dieser Form das Charakteristische der konkreten Verletzungsform zum Ausdruck kommt (...). Dem liegt die Erwägung zugrunde, daß eine in bestimmter Form begangene Verletzungshandlung nicht nur die Wiederbolung der genau identischen Verletzungsform vermuten läßt, sondern auch eine Vermutung für die Begehung zwar leicht abgewandelter, aber in ibrem Kern gleicher Handlungen begründet, wobei allerdings eine den Bestimmtheitsanforderungen genügende Grundlage für die Vollstreckung auch bei abweichenden Handlungsformen vorliegen muß.

Diesen Anforderungen wird der vom Berufungsgericht bestätigte Verbotsausspruch des Landgerichts nicht gerecht. Zu Recht beanstandet die Revision, daß das Verbot zu weit gefaßt ist. Aus dem Nichtvorhandensein der Clementinen, lediglich einem kleinen Teil des mit der angegriffenen Anzeige unter 'Obst und Gemüse' beworbenen Gesamtangebots der Beklagten, kann nicht die Vermutung abgeleitet werden, daß die Beklagte hinsichtlich sämtlicher anderer Waren und in allen Warenbereichen und Abteilungen ihres Unternehmens mangelhaft disponieren und insoweit wettbewerbswidrig werben werde. Das begehrte Verbot war deshalb auf Angebote der Produktgruppe Obst und Gemüse zu beschränken."

Heißt das, daß man bei fehlendem Notebook-Vorrat im Unterlassungsanspruch auf EDV-Waren generalisieren darf oder nicht? Die Frage stellen, heißt die Unklarheit der Entscheidungskriterien zu bemerken. Parallelisiert man „Clementinen $\rightarrow$ Obst und Gemüse -> Lebensmittel” mit „Notebooks -> EDV-Waren”, so kommt man eher zu der Einschätzung, daß ein Unterlassungsanspruch allenfalls für eine Teilmenge der EDV-Waren gegeben sein könnte. Das KG entwickelt im Kontrast dazu eine Homogenitäts=/Inhomogenitätstheorie bezogen auf Warenangebote, die so der BGI I-Entscheidung nicht zu entnehmen ist: Bei inhomogenem Warenangebot („Clementinen-Fall”: Frischwaren vs. sonstige Lebensmittel) kommt eine Generalisierung nur innerhalb des vom Vorratsmangel betroffenen Bereichs in Frage, bei homogenem Warenangebot ist eine stärkere Generalisierung erlaubt. Nur: Welches Kriterium bestimmt über die Homogenität eines Warenangebots? Wir wissen jetzt, wic das Kammergericht es im Falle von Notebooks und EDV-Waren diesbezüglich hält - die genaue Methode kennen wir indessen nicht.

Maximilian Herberger
. 